\title{
Trans-Splicing-Mediated Improvement in a Severe Mouse Model of Spinal Muscular Atrophy
}

\author{
Tristan H. Coady and Christian L. Lorson \\ Department of Veterinary Pathobiology, Bond Life Sciences Center, University of Missouri, Columbia, Missouri 65211
}

\begin{abstract}
Spinal muscular atrophy is a leading genetic cause of infantile death and occurs in $\sim 1 / 6000$ live births. SMA is caused by the loss of Survival Motor Neuron-1 (SMN1), however, all patients retain at least one copy of a nearly identical gene called SMN2. While SMN2 and $S M N 1$ are comprised of identical coding sequences, the majority of SMN2 transcripts are alternatively spliced, encoding a truncated protein that is unstable and nonfunctional. Considerable effort has focused upon modulating the SMN2 alternative splicing event since this would produce more wild-type protein. Recently we reported the development of an optimized trans-splicing system that involved the coexpression of a SMN2 trans-splicing RNA and an antisense RNA that blocks a downstream splice site in SMN2 pre-mRNA. Here, we demonstrate that in vivo delivery of the optimized trans-splicing vector increases an important SMN-dependent activity, snRNP assembly, in disease-relevant tissue in the SMA mouse model. A single injection of the vector into the intracerebral-ventricular space in SMA neonates also lessens the severity of the SMA phenotype in a severe SMA mouse model, extending survival $\sim 70 \%$. Collectively, these results provide the first in vivo demonstration that SMN2 trans-splicing leads to a lessening of the severity of the SMA phenotype and provide evidence for the power of this strategy for reprogramming genetic diseases at the pre-mRNA level.
\end{abstract}

\section{Introduction}

Spinal muscular atrophy (SMA), a neurodegenerative disorder, is caused by homozygous loss of survival motor neuron 1 (SMN1) and is a leading genetic cause of infantile death (Crawford and Pardo, 1996; Oskoui et al., 2007). The human genome contains SMN1 and SMN2 genes and while these genes are nearly identical, only SMN1 serves as the SMA-determining gene (Lefebvre et al., 1995; Rochette et al., 2001). The critical distinction between the SMN1 and SMN2 occurs at the RNA processing level: SMN1 produces primarily full-length transcripts, while SMN2 produces an alternatively spliced isoform lacking the final coding exon (Lefebvre et al., 1995). A single silent C to $\mathrm{T}$ nonpolymorphic nucleotide difference is responsible for disrupting a critical splice enhancer element in SMN2 exon 7 (Lorson et al., 1999; Cartegni and Krainer, 2002). SMN2 is retained in all SMA patients and is an ideal target for SMA therapeutic development (Sumner, 2006). The presence of SMN2 and the fact that SMA is monogenic have allowed progress in a number of therapeutic avenues. In addition to the identification and development of small molecules that stimulate promote exon 7 inclusion and/or fulllength SMN2 expression, RNA modalities such as antisense oligonucleotides (ASO), TOES/bifunctional RNAs, and trans-splicing RNAs have shown promise in cell-based and animal models of SMA (Lim and Hertel, 2001; Cartegni and Krainer, 2003; Skordis et al., 2003; Madocsai et al., 2005; Baughan et al., 2006, 2009; Singh et al., 2006, 2009; Coady et al., 2007, 2008; Hua et al., 2007, 2008; Marquis et al.,

Received Sept. 10, 2009; revised 0ct. 13, 2009; accepted Nov. 11, 2009.

This work was supported by grants from the National Institutes of Health (R01 HD054413; R01 HS41584). We thank John Marston for expert technical assistance.

Correspondence should be addressed to Dr. Christian L. Lorson, Department of Veterinary Pathobiology, Life Sciences Center, Room 471G, University of Missouri, Columbia, M0 65211. E-mail: Iorsonc@missouri.edu. DOI:10.1523/JNEUROSCI.4489-09.2010

Copyright $\odot 2010$ the authors $\quad 0270-6474 / 10 / 300126-05 \$ 15.00 / 0$
2007, 2009; Dickson et al., 2008; DiMatteo et al., 2008; Meyer et al., 2009; Williams et al., 2009).

Trans-splicing is a natural, albeit relatively uncommon process in mammals (Wood et al., 2007). Trans-splicing has recently been envisioned as a potential therapeutic intervention for a variety of genetic diseases, including spinal muscular atrophy, cystic fibrosis, hyper-IgM X-linked immunodeficiency, hemophilia A, Alzheimer's disease, and epidermolysis bullosa simplex with muscular dystrophy (Garcia-Blanco, 2003, 2004; RodriguezMartin et al., 2005; Coady et al., 2007; Zayed et al., 2007; Coady et al., 2008; Hengge, 2008; Wally et al., 2008). Unlike normal premRNA splicing that occurs within a single pre-mRNA substrate, trans-splicing occurs between two different molecules: (1) the mutant endogenous RNA and (2) the exogenous therapeutic RNA that provides the correct RNA sequence via a trans-splicing event. An important benefit to trans-splicing is that it maintains the native expression patterns since trans-splicing relies directly on endogenous mRNA expression.

Recently, we developed a strategy to increase trans-splicing efficiency by blocking the downstream splice site in cis-with an antisense RNA molecule (Coady et al., 2008). To examine the activity of trans-splicing in a severe model of SMA, plasmids expressing the SMN trans-splicing RNA and antisense RNA were transfected in vivo into the severe SMA mouse model. The severe SMA mouse $\left(m S M N^{-1-}, h S M N 2^{+/+}\right)$phenotype is very severe, with early synaptic occupation defects and an average life span of 3-4 d (Monani et al., 2000; McGovern et al., 2008). Here, we demonstrate that SMN2 trans-splicing functionally restores in important SMN-dependent pathway, snRNP assembly, within the CNS and that the average life span of severe SMA mice is significantly extended following a single administration of the optimized trans-splicing vector. 

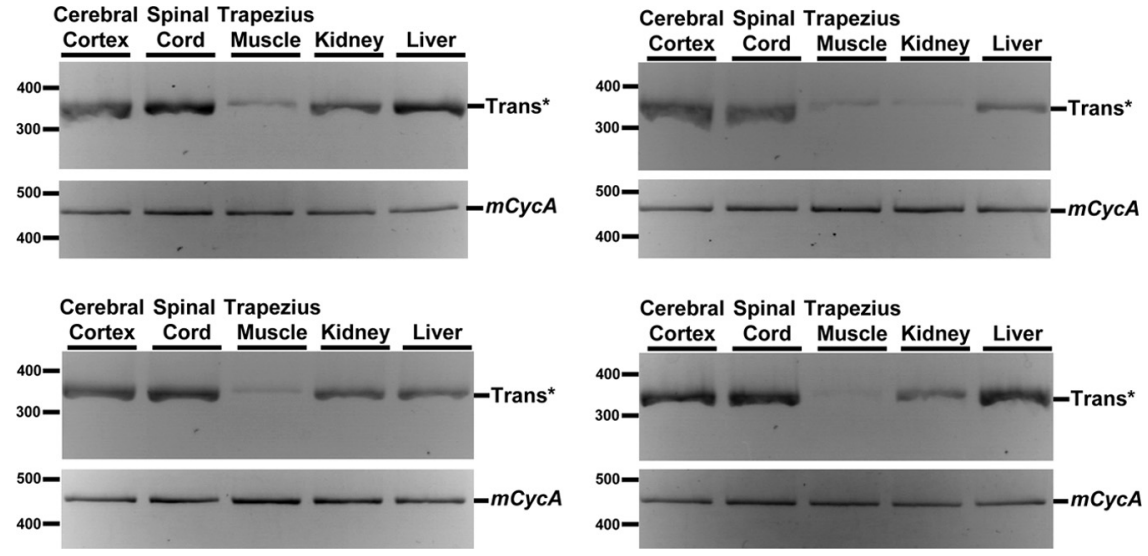

Figure 1. Trans-splicing detection in SMA tissues. Four representative severe SMA mice $\left(\mathrm{Smn}^{-1-} ; \mathrm{SMN2}{ }^{+/+}\right)$were assayed for trans-splicing using trans-splicing specific primers. The panel of gels shows RT-PCR results indicating the biodistribution of the trans-SMN RNA. Tissues were harvested $24 \mathrm{~h}$ posttransfection and mCycA was used as a positive control for loading and RT-PCR. Molecular weight markers are indicated on the left of each gel.

\section{Materials and Methods}

Plasmids. The ASO-tsRNA-expressing pMU3 plasmid and ptsRNA ${ }^{\mathrm{KO}}$ have been previously described (Coady et al., 2008). Plasmid was purified using a QIAgen kit and dissolved into phosphate buffered saline for injections.

Genotyping and mouse protocol. Animals were handled according to the University of Missouri Animal Care and Use Committee approved protocols. The $m S m n$ heterozygous mother would birth, counting as postnatal day 0 (PND0) and the neonates were genotyped within PND1. The genotyping, PCR conditions were $94^{\circ} \mathrm{C}$ denature for $2 \mathrm{~min}\left(94^{\circ} \mathrm{C}\right.$ denature for $15 \mathrm{~s}, 68^{\circ} \mathrm{C}$ anneal) for 29 cycles followed by an elongation at $68^{\circ} \mathrm{C}$ for $10 \mathrm{~min}$ (Coady et al., 2008). Results indicating SMA mice (null for Smn) were repeated in triplicate. Severe SMA mice were raised with 4 heterozygous siblings. Additional heterozygous and wild-type animals were culled within the first hours of PND1 in experimental cages. Each mouse examination was equal in its duration throughout the cohort with markings occurring on each mouse up to the last day of life. The mice were individually weighed at the same time each day to maintain feeding patterns and intraday metabolic differences. Dissections were as follows: the vertebral column was separated from the torso then divided into the cervical, thoracic, and lumbar (C-T-L) regions. The spinal cord was excised by performing two incisions in a posterior to anterior manner with a ventral approach. The ventral vertebral strip was then removed exposing the spinal cord white tissue. The C-T-L sections were further disrupted by shearing with a $1000 \mu$ l pipette tip. The resulting homogenates were aliquoted and immediately frozen.

$S M A$ trans-splicing RT-PCR. In vivo RT-PCR method is described previously (Coady et al., 2008). To remove possible genomic contaminants, DNase I-treated whole RNA stocks were seeded into RT reactions at 9.5 $\mu \mathrm{g}$ of total RNA. In vivo trans-splicing primer and reaction are previously described (Coady et al., 2008). The PCR amplification used a Roche High Fidelity kit. Negative controls were performed with no RT and no RNA to control for nonspecific amplification during the PCR.

Vector plasmid degradation and expression. Heterozygous mouse brain tissues were extracted and lysed in HiRT buffer. Total DNA was extracted with chloroform and precipitated with ethanol. The DNA reconstitution buffer was then treated with 1.5 units of RNase A to remove residual ribonucleic acids. DNA concentration was determined with the Bio-Rad slot blot vacuum. DNA $(5 \mu \mathrm{g})$ was used in Invitrogen Platinum Taq reactions with $1.25 \mathrm{U}$ of polymerase and $5 \mu \mathrm{l}$ of PCR buffer and $2.25 \mu \mathrm{l}$ of enhancer solution. The PCR cycle number was empirically determined to be 17 by the amplification of the positive control band and band appearance comparable to a 1:1 ratio with $1 \mu \mathrm{g}$ of standard in the $1 \mathrm{~kb}$ molecular marker. The positive-control heterozygous mouse was intracerebroventricularly injected on PND 1 and was harvested $24 \mathrm{~h}$ later. The brain was dissected out and the cerebellum and olfactory lobes excised leaving the cerebral cortex. This brain section contains the majority of the ventricle space and therefore the highest exposure to the vector. The cortex was further divided through the sagittal and then the coronal plane creating four sections. These were titled the anterior (A) and the posterior $(\mathrm{P})$ samples. The negative control heterozygous mouse was from a different litter injected with Mock solution described previously which contains no plasmid.

In vivo UsnRNP assembly. The procedure was adapted as previously described for tissuederived snRNP assembly assays (Gabanella et al., 2007). The lumbar section of the spine was isolated and passed through the tip of a $1000 \mu \mathrm{l}$ pipette tip to disperse the tissue in cold PBS. The fresh cells were counted on a hemocytometer then $4 \times 10^{6}$ cells aliquots placed into new tubes by spinning at $500 \mathrm{rpm}$ for $6 \mathrm{~min}$ in a cold centrifuge. The spinal cord pellets were resuspended evenly in RB-buffer and then Triton $\mathrm{X}-100$ was added to a final percentage of $0.1 \%$ to separate the cytoplasm from the nucleus. The cytoplasmic fractions were then quantitated using the Bradford/ Lowry method to calculate $25 \mu \mathrm{g}$ of total protein used in the assembly reactions.

\section{Results}

The determine whether in vivo increases in SMN protein in a severe mouse model of SMA could lessen the severity of the phenotype, an in vivo assessment of trans-splicing in the severe SMA model was initiated. This model lacks endogenous murine Smn but contains two genomic copies of the human SMN2 gene, and is therefore ideally suited for translational studies that target SMN2 alternative splicing. To determine the biodistribution and efficiency of trans-splicing in disease-relevant tissues, SMA pups were received intracerebral-ventricular injections with vector expressing the tsRNA and antisense RNA, pMU3. Tissues from four separate SMA animals were examined. $24 \mathrm{~h}$ after receiving a single injection, SMN trans-splicing was readily detectable within the CNS, as well as distal sites, including kidney and liver. To a lesser degree, trans-splicing was also detected within skeletal muscle (Fig. 1). It is possible that in neonatal animals, the blood brain barrier is more permeable, allowing greater levels of transport into the circulatory system, helping to explain the detectable levels of trans-splicing in distal sites. SMN trans-splicing was not detected in any tissue from untreated SMA pups or from a pMU3 derivative that lacked the tsRNA promoter (tsRNA ${ }^{\mathrm{KO}}$ ) (data not shown and supplemental Fig. 1, available at www.jneurosci.org as supplemental material). GFP expression derived from the tsRNA vector was monitored and was detected throughout the spinal column, consistent with the trans-splicing results (supplemental Fig. 2, available at www.jneurosci.org as supplemental material). Plasmid-derived GFP tissue expression correlated with the observed trans-splicing (data not shown). Based upon these results and a recent report that demonstrates that high levels of neuronal SMN significantly rescues the SMA phenotype (Gavrilina et al., 2008), subsequent experiments were focused upon the CNS.

$\mathrm{SMN}$ is a multifunctional protein, however, it is not clear which specific function is connected to the development of SMA. The best described biochemical function of SMN centers around the assembly of small nuclear ribonuclear protein complexes, snRNPs, which are the building blocks for the spliceosome. To determine whether SMA mice treated with the trans-splicing vector exhibited increased levels of snRNP assembly activity, protein 
A
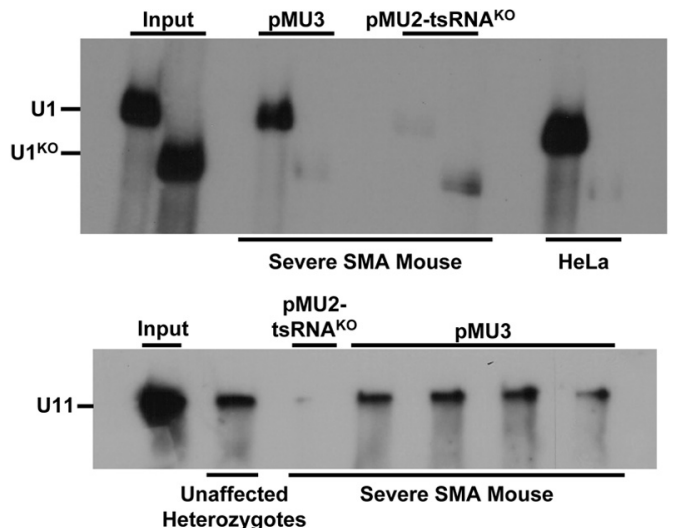

B Unaffected Heterozygotes SMA

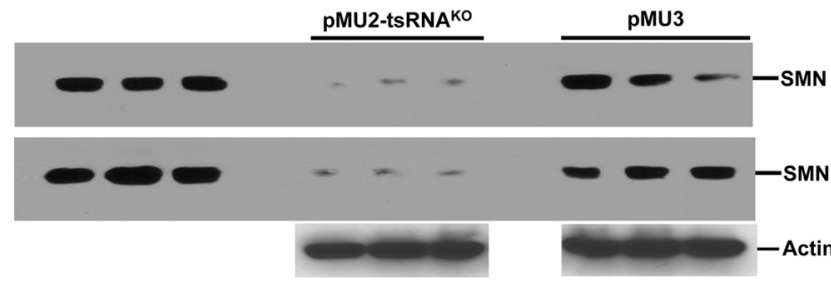

Figure 2. Trans-splicing mediated increases in functional SMN levels in vivo. $\boldsymbol{A}$, In vivo restoration of severe SMA neonatal UsnRNP assembly capacity is mediated by SMN trans-splicing RNA plasmid injections. U1 and U11 snRNAs were radiolabeled in vitro and incubated with the respective extracts. Y12 antibody was used to immunoprecipitate snRNP complexes from tissue extracts derived from treated SMA mice or unaffected heterozygote mice. $\mathrm{U}^{\mathrm{K} O}$ is a negative control RNA that lacks the Sm nucleation site. Mouse lumbar spinal cord sections were removed from the vertebrae and passed through a 25 gauge needle before the assembly process. The negative control SMA mouse was injected with pMU2-tsRNA ${ }^{\mathrm{KO}}$ plasmid. HeLa cell extract served as a positive control. Representative results from three independent experiments are shown. $\boldsymbol{B}$, Western blots of spinal cords from ASO-tsRNA-treated mice demonstrated increases in SMN protein. 10\% SDS-PAGE gels are shown developed with an anti-SMN monoclonal antibody. Six separate SMA mice were used to generate statistical significance between treatment and control transfections. Equal amounts of protein were loaded in each well as determined by Lowry assay.

extracts were generated from pMU3-treated and control-treated animals. Extracts from the lumbar spinal cord region were generated and incubated with radiolabeled U1 snRNA. As anticipated, HeLa cell extracts supported very high levels of snRNP assembly, consistent with the high levels of endogenous SMN protein (Fig. $2 A$ ). In pMU3-treated SMA extracts, snRNP assembly levels were dramatically elevated compared to control vectortreated extracts (Fig. 2A). U1 is a component of the major splicing pathway, however, defects in the assembly of minor splicing snRNP components have also been identified in SMA animals (Gabanella et al., 2007). To examine SMN activity in this alternative pathway, snRNP assembly on minor pathway snRNA, U11, was examined in similar extracts (Fig. 2A, bottom panel). Consistent with the increased activity observed in the U1dependent assays, snRNP assembly was elevated using the U11 snRNA template (Fig. $2 A$, bottom panel). SMN protein levels were examined by Western blot in lumbar spinal cord extracts to correlate the apparent increase in SMN-dependent snRNP assembly activity with increased SMN protein levels (Fig. 2 B; supplemental Fig. 3, available at www.jneurosci.org as supplemental material). Consistent with the increased snRNP assembly activity, steady-state SMN protein levels were increased in transsplicing-treated tissues (Fig. 2B). While it is not clear that snRNP

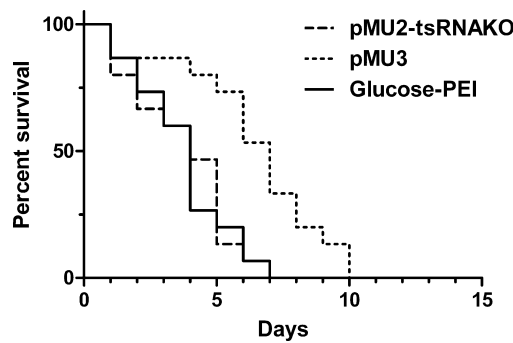

Figure 3. Intracerebroventricular delivery of optimized trans-splicing vector extends average life span in a severe model of SMA. Neonatal pups were injected with a mixture of PEI/ Glucose and plasmid vector via a single intracerebral-ventricular injection. The pMU3 intracerebroventricular injection produced an approximate $\sim 70 \%$ increase in SMA mouse survival. Kaplan-Meier curve depicts negative controls vehicle (Glucose/PEI) and tsRNA ${ }^{\mathrm{K} 0}$ dying at a maximum $7 \mathrm{~d} . n=15$ mice for each group. $p<0.001$.

assembly is the critical function underlying SMA development, these results demonstrate that SMN levels can be significantly increased in disease-relevant tissue and that this increase correlates with elevated snRNP assembly levels.

To examine the potential for physiological changes in the SMA mice we used a more severe mouse model for two reasons: (1) The severe mouse model experimental duration is much shorter than the $\Delta 7$ mice and the ability to deliver via repeated intracerebroventricular injection is limited; (2) the severity of the model could potentially be a benefit because a small change would be measurable rather than in a less severe model. To determine whether the previous biochemical and molecular increases in SMN splicing and protein levels resulted in detectable changes in the SMA phenotype in SMA mice, weight and life span data were collected following a single intracerebral-ventricular injection at postnatal day 1 . Three cohorts consisting of 15 SMA pups per group were examined: the trans-splicing vector (pMU3); an early generation trans-splicing vector that lacked the transsplicing RNA promoter; and vehicle alone (Glucose-PEI). As previously published, this model of SMA is very severe, resulting in low birth weights and death for the majority of the vehicle-treated animals in $<5 \mathrm{~d}$ (Monani et al., 2000). Similarly, the control vector lacking the promoter resulted in nearly identical data, with no animal living past $7 \mathrm{~d}$, with an average life span of $\sim 4 \mathrm{~d}$ (Fig. 3). SMA pups transiently transfected with the trans-splicing vector, however, had an average life span of $\sim 7 \mathrm{~d}$, significantly longer than the control cohorts (Mantel-Cox test, $P=0.001$ ) (Fig. 3). Additionally, the longest lived animals reached 9 and $10 \mathrm{~d}$ (Fig. 3). Weight differences were not observed between untreated and treated SMA groups, suggesting that this is still an extremely severe model of neurodegeneration (data not shown). Even though this model is exceptionally severe, collectively, these results demonstrate that increases in functional SMN protein can be achieved in vivo and that these increases can significantly alter the disease phenotype.

\section{Discussion}

SMN2 has been envisioned as an outstanding target for therapeutic intervention since it is retained in all SMA patients and it has the potential to encode a fully functional SMN protein. Strategies that redirect the SMN2 alternative splicing event have been particularly intriguing for RNA-based therapeutics, including a variety of antisense oligonucleotides as well as trans-splicing. Recent reports highlight the potential application of RNA-based therapeutics designed to increase full-length expression from SMN2, including the delivery of ASOs directly to the CNS of SMA pups 
through intracerebroventricular injection (Dickson et al., 2008; Baughan et al., 2009; Williams et al., 2009). In this report we demonstrate that following intracerebroventricular administration of an optimized trans-splicing vector, in vivo trans-splicing of the SMN2 gene results in increased levels of functional SMN, consistent with a significant increase in average life span for transsplicing treated SMA pups.

We chose to measure snRNP assembly as a means of monitoring SMN functionality. The role of SMN in snRNP assembly has been extremely well defined and analyzed at a very high-resolution level from a biochemical perspective (Pellizzoni, 2007). While the exact role of SMN in major versus minor splicing pathways remains unclear, simply using the snRNP assembly assay in spinal cord extracts provides a tractable biochemical assay to monitor functional SMN levels. It remains a possibility that an alternative function such as axonal transport of mRNAs is the primary cause of SMA development; however, a well defined assay system for this type of activity has not been described and we are making a logical assumption that full-length SMN restores or significantly increases all SMN-dependent deficiencies.

While these are clearly proof-of-concept experiments, the experiments raise in intriguing question in SMA: when can SMA therapeutics be delivered to elicit a response? Two SMA animal models have been the focus of therapeutic analysis: the severe model used in this report and the less severe " $\Delta 7$ " SMA mouse model. Intraperitoneal injections of the HDAC inhibitor trichostatin A (TSA) in the $\Delta 7$ model significantly extended survival (Narver et al., 2008), suggesting that this model may not be intractable and can be fully rescued by more potent SMN activators or gene replacement strategies. To date, the extreme disease progression observed with the "severe" model suggests that prenatal deficiencies exist. While further studies will be required to draw accurate conclusions, the severe model still serves as an important proof-of-concept model that is exceptionally simple at the genetic level and allows that rapid analysis of in vivo applications such as trans-splicing.

Intracerebroventricular delivery provides a robust means to directly deliver SMA therapeutics to disease-relevant tissues within the CNS. As the benefits for oligonucleotide-mediated and vector-mediated strategies are weighed, sustained expression will likely be an important factor. It is likely that SMN will continue to be required throughout life; therefore, either a continuous pump or longer-term viral vector will likely move closer toward clinical reality. These results provide the first in vivo proof-of-concept evidence that SMN trans-splicing can lessen the severity of the SMA phenotype and provide a platform to move forward into a viral vector system that can provide sustained and robust expression of functional SMN.

\section{References}

Baughan T, Shababi M, Coady TH, Dickson AM, Tullis GE, Lorson CL (2006) Stimulating full-length SMN2 expression by delivering bifunctional RNAs via a viral vector. Mol Ther 14:54-62.

Baughan TD, Dickson A, Osman EY, Lorson CL (2009) Delivery of bifunctional RNAs that target an intronic repressor and increase SMN levels in an animal model of spinal muscular atrophy. Hum Mol Genet $18: 1600-1611$.

Cartegni L, Krainer AR (2002) Disruption of an SF2/ASF-dependent exonic splicing enhancer in SMN2 causes spinal muscular atrophy in the absence of SMN1. Nat Genet 30:377-384.

Cartegni L, Krainer AR (2003) Correction of disease-associated exon skipping by synthetic exon-specific activators. Nat Struct Biol 10:120-125.

Coady TH, Shababi M, Tullis GE, Lorson CL (2007) Restoration of SMN function: delivery of a trans-splicing RNA re-directs SMN2 pre-mRNA splicing. Mol Ther 15:1471-1478.
Coady TH, Baughan TD, Shababi M, Passini MA, Lorson CL (2008) Development of a single vector system that enhances trans-splicing of SMN2 transcripts. PLoS ONE 3:e3468.

Crawford TO, Pardo CA (1996) The neurobiology of childhood spinal muscular atrophy. Neurobiol Dis 3:97-110.

Dickson A, Osman E, Lorson CL (2008) A negatively-acting bifunctional RNA increases survival motor neuron in vitro and in vivo. Hum Gene Ther 19:1307-1315.

DiMatteo D, Callahan S, Kmiec EB (2008) Genetic conversion of an SMN2 gene to SMN1: a novel approach to the treatment of spinal muscular atrophy. Exp Cell Res 314:878-886.

Gabanella F, Butchbach ME, Saieva L, Carissimi C, Burghes AH, Pellizzoni L (2007) Ribonucleoprotein assembly defects correlate with spinal muscular atrophy severity and preferentially affect a subset of spliceosomal snRNPs. PLoS ONE 2:e921.

Garcia-Blanco MA (2003) Messenger RNA reprogramming by spliceosomemediated RNA trans-splicing. J Clin Invest 112:474-480.

Garcia-Blanco MA, Baraniak AP, Lasda EL (2004) Alternative splicing in disease and therapy. Nat Biotechnol 22:535-546.

Gavrilina TO, McGovern VL, Workman E, Crawford TO, Gogliotti RG, DiDonato CJ, Monani UR, Morris GE, Burghes AH (2008) Neuronal SMN expression corrects spinal muscular atrophy in severe SMA mice while muscle-specific SMN expression has no phenotypic effect. Hum Mol Genet 17:1063-1075.

Hengge UR (2008) SMaRT technology enables gene expression repair in skin gene therapy. J Invest Dermatol 128:499-500.

Hua Y, Vickers TA, Baker BF, Bennett CF, Krainer AR (2007) Enhancement of SMN2 exon 7 inclusion by antisense oligonucleotides targeting the exon. PLoS Biol 5:e73.

Hua Y, Vickers TA, Okunola HL, Bennett CF, Krainer AR (2008) Antisense masking of an hnRNP A1/A2 intronic splicing silencer corrects SMN2 splicing in transgenic mice. Am J Hum Genet 82:834-848.

Lefebvre S, Bürglen L, Reboullet S, Clermont O, Burlet P, Viollet L, Benichou B, Cruaud C, Millasseau P, Zeviani M, Paslier DL, Frezal J, Cohen D, Weissenbach J, Munnich A, Melki J (1995) Identification and characterization of a spinal muscular atrophy- determining gene. Cell 80:155-165.

Lim SR, Hertel KJ (2001) Modulation of survival motor neuron pre-mRNA splicing by inhibition of alternative $3^{\prime}$ splice site pairing. J Biol Chem 276:45476-45483.

Lorson CL, Hahnen E, Androphy EJ, Wirth B (1999) A single nucleotide in the SMN gene regulates splicing and is responsible for spinal muscular atrophy. Proc Natl Acad Sci U S A 96:6307-6311.

Madocsai C, Lim SR, Geib T, Lam BJ, Hertel KJ (2005) Correction of SMN2 Pre-mRNA splicing by antisense U7 small nuclear RNAs. Mol Ther 12:1013-1022.

Marquis J, Meyer K, Angehrn L, Kämpfer SS, Rothen-Rutishauser B, Schümperli D (2007) Spinal muscular atrophy: SMN2 pre-mRNA splicing corrected by a U7 snRNA derivative carrying a splicing enhancer sequence. Mol Ther 15:1479-1486.

Marquis J, Kämpfer SS, Angehrn L, Schümperli D (2009) Doxycyclinecontrolled splicing modulation by regulated antisense U7 snRNA expression cassettes. Gene Ther 16:70-77.

McGovern VL, Gavrilina TO, Beattie CE, Burghes AH (2008) Embryonic motor axon development in the severe SMA mouse. Hum Mol Genet 17:2900-2909.

Meyer K, Marquis J, Trüb J, Nlend Nlend R, Verp S, Ruepp MD, Imboden H, Barde I, Trono D, Schümperli D (2009) Rescue of a severe mouse model for spinal muscular atrophy by U7 snRNA-mediated splicing modulation. Hum Mol Genet 18:546-555.

Monani UR, Sendtner M, Coovert DD, Parsons DW, Andreassi C, Le TT, Jablonka S, Schrank B, Rossol W, Prior TW, Morris GE, Burghes AH (2000) The human centromeric survival motor neuron gene (SMN2) rescues embryonic lethality in $\operatorname{Smn}(-/-)$ mice and results in a mouse with spinal muscular atrophy. Hum Mol Genet 9:333-339.

Narver HL, Kong L, Burnett BG, Choe DW, Bosch-Marcé M, Taye AA, Eckhaus MA, Sumner CJ (2008) Sustained improvement of spinal muscular atrophy mice treated with trichostatin a plus nutrition. Ann Neurol 64:465-470.

Oskoui M, Levy G, Garland CJ, Gray JM, O’Hagen J, De Vivo DC, Kaufmann P (2007) The changing natural history of spinal muscular atrophy type 1. Neurology 69:1931-1936. 
Pellizzoni L (2007) Chaperoning ribonucleoprotein biogenesis in health and disease. ЕMBO Rep 8:340-345.

Rochette CF, Gilbert N, Simard LR (2001) SMN gene duplication and the emergence of the SMN2 gene occurred in distinct hominids: SMN2 is unique to Homo sapiens. Hum Genet 108:255-266.

Rodriguez-Martin T, Garcia-Blanco MA, Mansfield SG, Grover AC, Hutton M, Yu Q, Zhou J, Anderton BH, Gallo JM (2005) Reprogramming of tau alternative splicing by spliceosome-mediated RNA trans-splicing: implications for tauopathies. Proc Natl Acad Sci U S A 102:15659-15664.

Singh NK, Singh NN, Androphy EJ, Singh RN (2006) Splicing of a critical exon of human Survival Motor Neuron is regulated by a unique silencer element located in the last intron. Mol Cell Biol 26:1333-1346.

Singh NN, Shishimorova M, Cao LC, Gangwani L, Singh RN (2009) A short antisense oligonucleotide masking a unique intronic motif prevents skipping of a critical exon in spinal muscular atrophy. RNA Biol 6:341-350.

Skordis LA, Dunckley MG, Yue B, Eperon IC, Muntoni F (2003) Bifunctional antisense oligonucleotides provide a trans-acting splicing enhancer that stimulates SMN2 gene expression in patient fibroblasts. Proc Natl Acad Sci U S A 100:4114-4119.

Sumner CJ (2006) Therapeutics development for spinal muscular atrophy. NeuroRx 3:235-245.

Wally V, Klausegger A, Koller U, Lochmüller H, Krause S, Wiche G, Mitchell LG, Hintner H, Bauer JW (2008) $5^{\prime}$ trans-splicing repair of the PLEC1 gene. J Invest Dermatol 128:568-574.

Williams JH, Schray RC, Patterson CA, Ayitey SO, Tallent MK, Lutz GJ (2009) Oligonucleotide-mediated survival of motor neuron protein expression in CNS improves phenotype in a mouse model of spinal muscular atrophy. J Neurosci 29:7633-7638.

Wood M, Yin H, McClorey G (2007) Modulating the expression of disease genes with RNA-based therapy. PLoS Genet 3:e109.

Zayed H, Xia L, Yerich A, Yant SR, Kay MA, Puttaraju M, McGarrity GJ, Wiest DL, McIvor RS, Tolar J, Blazar BR (2007) Correction of DNA protein kinase deficiency by spliceosome-mediated RNA trans-splicing and sleeping beauty transposon delivery. Mol Ther 15:1273-1279. 